target analyses of chemotherapy-naive muscle-invasive bladder cancer with a molecular screening. Oncotarget 9:25935-25945

59. Rosenberg JE, Hoffman-Censits J, Powles T et al (2016) Atezolizumab in patients with locally advanced and metastatic urothelial carcinoma who have progressed following treatment with platinum-based chemotherapy: a single-arm, multicentre, phase 2 trial. Lancet 387:1909-1920

60. Salvi S, Casadio V, Conteduca V et al (2015) Circulating cell-free AR and CYP17A1 copy number variations may associate with outcome of metastatic castration-resistant prostate cancer patients treated with abiraterone. $\mathrm{Br} \mathrm{J}$ Cancer 112:1717-1724

61. Schildhaus HU (2018) Predictive value of PD-L1 diagnostics. Pathologe 39:498-519

62. Schuler M, Nogova L, Heidenreich A et al (2017) 859PAnti-tumor activity of the pan-FGFR inhibitor rogaratinib in patients with advanced urothelial carcinomas selected based on tumor FGFR mRNA expression levels. Ann Oncol 28:v295. https://doi. org/10.1093/annonc/mdx371

63. Seiler R, Erho AHDN et al (2017) Impact of molecular subtypes in muscle-invasive bladder cancer on predicting response and survival after neoadjuvant chemotherapy. Eur Urol 72:544-554

64. Siefker-Radtke A, Necchi A, ParkSH et al (2018) First results from the primary analysis population of the phase 2 study of erdafitinib (ERDA; JNJ-42756493) in patients (pts) with metastatic or unresectable urothelial carcinoma ( $\mathrm{mUC}$ ) and FGFR alterations (FGFRalt). JClin Oncol 36(15_suppl):4503

65. Sjodahl G, Eriksson P, Liedberg F et al (2017) Molecular classification of urothelial carcinoma: global mRNA classification versus tumour-cell phenotype classification. JPathol 242:113-125

66. Sjodahl G, Lauss M, Lovgren K et al (2012) A molecular taxonomy for urothelial carcinoma. Clin Cancer Res 18:3377-3386

67. Szarvas T, Olah C, Reis H (2019) Neoadjuvant cisplatin-based chemotherapy in "primary" and "secondary" muscle-invasive bladder cancer-is it a surrogate for molecular subtypes? TransI Cancer Res. https://doi.org/10.21037/tcr.2019.01.05

68. Tretiakova $M$, Fulton $R$, Kocherginsky $M$ et al (2018) Concordance study of PD-L1 expression in primary and metastatic bladder carcinomas: comparison of four commonly used antibodies and RNA expression. Mod Pathol 31:623-632

69. Turkbey B, Rosenkrantz AB, Haider MA et al (2019) Prostate imaging reporting and data system version 2.1: 2019 update of prostate imaging reporting and data system version 2. Eur Urol. https://doi.org/10.1016/j.eururo.2019.02.033

70. Turney A (2019) FDA approves first targeted therapy for metastatic bladder cancer. In: U.S. Food and Drug Administration. https://www.fda.gov/ NewsEvents/Newsroom/PressAnnouncements/ ucm635906.htm. Zugegriffen: 29. Januar 2019

71. Udager AM, Mcdaniel AS, Hovelson DH et al (2018) Frequent PD-L1 protein expression and molecular correlates in urinary bladder squamous cell carcinoma. Eur Urol 74:529-531

72. Van Allen EM, Mouw KW, Kim P et al (2014) Somatic ERCC2 mutations correlate with cisplatin sensitivity in muscle-invasive urothelial carcinoma. Cancer Discov 4:1140-1153

73. Voss MH, Reising A, Cheng $Y$ et al (2018) Genomically annotated risk model for advanced renal-cell carcinoma: a retrospective cohort study. Lancet Oncol 19:1688-1698

74. Yarchoan M, Johnson BA 3rd, Lutz ER et al (2017) Targeting neoantigens to augment antitumour immunity. Nat Rev Cancer 17:209-222

\title{
Erratum
}

Pathologe $2019 \cdot 40: 275$

https://doi.org/10.1007/s00292-019-0582-7

C) Springer Medizin Verlag GmbH, ein Teil von Springer Nature 2019

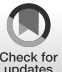

\section{H.-U. Schildhaus}

${ }^{1}$ Institut für Pathologie, Universitätsklinikum Essen (AöR), Essen, Deutschland

\section{Erratum zu: Der prädiktive Wert der PD-L1-Diagnostik}

\author{
Erratum zu: \\ Pathologe 2018 \\ https://doi.org/10.1007/s00292-018- \\ 0507-x
}

Bei der Publikation „Der prädiktive Wert der PD-L1-Diagnostik" fehlt in der dritten Spalte von Tab. 5 am Ende der Formeln sowie in der Legende von Abb. 7 bei der Berechnung des CPS $(50+250) / 350 \times 100=85$ jeweils der Faktor „ $\times 100$ “. Des Weiteren muss der letzte Satz des ersten Absatzes auf S. 502 durch die Angabe eines Antikörpers ergänzt werden und lautet vollständig: „Es konnte in klinischen Studien gezeigt werden, dass Tumoren mit einer hohen Mutationslast (für bestimmte Lungenkarzinome gilt hier 10 Mutationen pro Megabase als Schwellenwert) auf eine Behandlung mit dem PD-1Inhibitor Nivolumab in Kombination mit Ipilimumab ansprechen [10]“. Wir bitten, die Korrekturen zu beachten.

\section{Korrespondenzadresse}

Prof. Dr. H.-U. Schildhaus

Institut für Pathologie, Universitätsklinikum Essen (AöR)

Hufelandstraße 55, 45147 Essen, Deutschland Hans-Ulrich.Schildhaus@uk-essen.de
Die Online-Version des Originalartikels ist unter https://doi.org/10.1007/s00292-018-0507-xzu finden. 